\title{
Reproducibility and uptake time dependency of volume-based parameters on FDG-PET for lung cancer
}

Tomoka Kitao ${ }^{1,2}$, Kenji Hirata ${ }^{2 *}$, Katsumi Shima ${ }^{1}$, Takashi Hayashi ${ }^{1}$, Mitsunori Sekizawa', Toshiki Takei ${ }^{3}$, Wataru Ichimura ${ }^{3}$, Masao Harada ${ }^{4}$, Keishi Kondo ${ }^{5}$ and Nagara Tamaki ${ }^{2}$

\begin{abstract}
Background: Volume-based parameters, such as metabolic tumor volume (MTV) and total lesion glycolysis (TLG), on F-18 fluorodeoxyglucose (FDG) positron emission tomography (PET) are useful for predicting treatment response in nonsmall cell lung cancer (NSCLC). We aimed to examine intra- and inter-operator reproducibility to measure the MTV and TLG, and to estimate their dependency on the uptake time.

Methods: Fifty NSCLC patients underwent preoperative FDG-PET. After an injection of FDG, the whole body was scanned twice: at the early phase $(61.4 \pm 2.8 \mathrm{~min})$ and delayed phase $(117.7 \pm 1.6 \mathrm{~min})$. Two operators independently defined the tumor boundary using three different delineation methods: (1) the absolute SUV threshold method (MTV $v_{p}$ and $\left.T L G_{p} ; p=2.0,2.5,3.0,3.5\right)$, (2) the fixed\% SUVmax threshold method (MTV $q \%$ and $T L G_{q \% ;} q=35,40,45$ ), and (3) the adaptive region-growing method (MTV ${ }_{A R G}$ and $T L G_{A R G}$ ). Parameters were compared between operators and between phases.

Results: Both the intra- and inter-operator reproducibility were high for all parameters using any method (intra-class correlation $>0.99$ each). $M T V_{3.0}$ and $M T V_{3.5}$ resulted in a significant increase from the early to delayed phase $\left(P<0.05\right.$ for both), whereas $\mathrm{MTV}_{2.0}$ and $\mathrm{MTV}_{2.5}$ neither increased nor decreased $(P=$ n.s.). All of the $\operatorname{MTV}_{\mathrm{q} \%}$ values significantly decreased over time $(P<0.01)$, whereas MTV ARG and TLG with any delineation method increased significantly $(P<0.05)$.

Conclusions: High reproducibility of MTV and TLG was obtained by all of the methods used. MTV 2.0 and $M_{T V} V_{2.5}$ were the least sensitive to uptake time, and may be good alternatives when we compare images acquired with different uptake times, although applying constant uptake time is important for volume measurement.
\end{abstract}

Keywords: Lung cancer, Reproducibility, Metabolic tumor volume, Total lesion glycolysis, FDG-PET

\section{Background}

Positron emission tomography (PET) using F-18 fluorodeoxyglucose (FDG) has been an essential diagnostic tool in oncology [1-3]. FDG-PET generates functional images that contribute to clinical diagnoses and treatment planning complementarily with anatomical modalities such as computed tomography (CT) and magnetic

\footnotetext{
* Correspondence: khirata@med.hokudai.ac.jp

2Department of Nuclear Medicine, Graduate School of Medicine, Hokkaido University, Kita 15, Nishi 7, Kita-Ku, Sapporo, Hokkaido 060-8638, Japan Full list of author information is available at the end of the article
}

resonance imaging (MRI). PET is also characterized by high quantitative performance [4-6]. In most clinical settings, FDG-PET images were assessed semi-quantitatively using the standardized uptake value (SUV), which commonly represents the radioactivity concentration per unit volume of tissue normalized to the injected dosage and body weight [7]. The maximum of the SUV (SUVmax) within the tumor has been used most frequently to express the intensity of FDG uptake in the tumor because of its simplicity and high reproducibility [8-12]. However, the SUVmax has several problems. Because the SUVmax represents just a single voxel (normally $<0.1 \mathrm{ml}$ ) and not 
the entire tumor metabolism, it is sensitive to statistical noise of the image [13]. In recent years, the use of the SUVpeak has been preferred [13]. The definition of SUVpeak remains to be standardized, but usually calculated by averaging SUV within a 1- $\mathrm{ml}$ sphere (12 $\mathrm{mm}$ in diameter) around the voxel showing highest intensity voxel. The SUVpeak is less sensitive to image noise but suffers from the same problem as SUVmax still reflects a small part of the tumor $[14,15]$.

In this context, the metabolic tumor volume (MTV) and total lesion glycolysis (TLG) has been recently used as indices of the whole tumor FDG uptake. The MTV is defined as the volume of tumor determined on an FDG-PET image using a certain threshold. Once MTV is determined, the SUVmean can be defined as the averaged SUV within MTV. TLG is the product of the MTV and the SUVmean. These indicators reflect the activity of the glucose metabolism in the entire tumor. The clinical usefulness of these indicators (e.g., prognosis and treatment response) has been demonstrated in many cancers such as lung [16, 17], head-and-neck [18-20], and gynecological cancer [21, 22].

Calculating the MTV and TLG requires tumor contouring on the PET image. Many methods have been reported to determine the contour [23-31], and among them, manual contouring, the absolute SUV threshold method, and relative SUV threshold methods have been used widely. With the manual contouring method, the tumor boundary is determined based on an operator's visual inspection. This operator-dependent method suffers from reproducibility and is affected by the window level and color scale. It also takes a long time to apply this manual operation to all of the images containing tumors. Other methods have thus been developed to reduce the effects from display conditions or operators.

There is no doubt that the SUVmax has high intraand inter-operator reproducibility, but the reproducibility of MTV and TLG still needs to be assessed. In the present study, we examined intra-operator reproducibility (i.e., the same operator analyzes the same image twice) and inter-operator reproducibility (i.e., two operators analyze the same image independently). In addition, considering possible effects of uptake time after the FDG administration on the MTV and TLG, we acquired PET images twice after a single injection (at 60 and $120 \mathrm{~min}$ ), and we compared the MTV and TLG between these images. We applied different delineation methods that are widely used. Thus, in this study, we aimed to evaluate (1) intraoperator reproducibility, (2) inter-operator reproducibility, and (3) the effect of uptake time differences on volume-based parameters.

\section{Methods}

\section{Study subjects}

All procedures performed in studies involving human participants were in accordance with the ethical standards of the institutional and/or national research committee and with the 1964 Helsinki declaration and its later amendments or comparable ethical standards. The institutional ethics committee of Hokkaido Cancer Center approved this retrospective study. Informed consent was waived from individual participants in the retrospective study according to the committee. Patient records/information was anonymized and de-identified prior to analysis. From our hospital information system, we found a total of 52 patients who underwent FDGPET for an examination of lung nodules before treatment at the National Hospital Organization Hokkaido Cancer Center between December 2010 and March 2012. One patient was suspected of having metastatic lung tumor from breast cancer, and another patient did not complete the scanning because of severe pain. Thus, we included 50 patients (27 males; age, $70.2 \pm 10.1$ years old) whose lung nodules were visualized by FDG-PET and whose nodule(s) were pathologically confirmed as non-small cell lung cancer (NSCLC). The patient characteristics are shown in Table 1. Briefly, body weight was $56.0 \pm 9.0 \mathrm{~kg}$ (range 39-87 kg); tumor existed in the upper lobe $(N=30)$, the middle lobe $(N=4)$, or the lower lobe $(N=16)$ of the lung; pathological diagnosis was adenocarcinoma $(N=28)$, squamous cell carcinoma $(N=14)$, or others $(N=8)$; cancer stage was IA $(N=12)$, IB $(N=6)$, IIA $(N=10)$, IIB $(N=3)$, IIIA $(N=8)$, IIIB $(N=5)$ or IV $(N=6)$ based on the American Joint Committee on Cancer (AJCC) TNM system.

\section{Image acquisition and reconstruction}

All of the clinical FDG-PET studies were performed with an Eminence SET-3000G PET scanner (Shimadzu, Kyoto, Japan). All of the patients fasted for at least $6 \mathrm{~h}$ before the injection of FDG $(224 \pm 54 \mathrm{MBq}$, range $142-294 \mathrm{MBq} ; 4.0 \pm 0.9 \mathrm{MBq} / \mathrm{kg}$, range $2.5-$ 6.4 $\mathrm{MBq} / \mathrm{kg}$ ). The blood glucose level was $100 \pm$ $19 \mathrm{mg} / \mathrm{dl}$. The images were scanned twice for each study: early scanning at $61.4 \pm 2.8 \mathrm{~min}$ (range 58 $67 \mathrm{~min}$ ) and delayed scanning at $117.7 \pm 1.6 \mathrm{~min}$ (range 114-121 $\mathrm{min}$ ). The transaxial field of view was $512 \mathrm{~mm}$ in diameter. Three-dimensional emission scanning was performed in a continuous bedmovement manner $(0.8-0.9 \mathrm{~mm} / \mathrm{s})$. Transmission scanning was performed with a ${ }^{137} \mathrm{Cs}$ external source to correct for attenuation.

Images were reconstructed with a block-iterative algorithm named 'dynamic row-action maximum likelihood algorithm (DRAMA), modified from the rowaction maximum likelihood algorithm (RAMLA) [32]. 
Table 1 Patient characteristics

\begin{tabular}{|c|c|c|c|c|c|c|c|c|}
\hline Case & Age range (y) & Weight (kg) & Dosage (MBq) & Dosage/Weight (MBq/kg) & Tumor location & Pathology ${ }^{a}$ & TNM classification & Stage \\
\hline 1 & $80-89$ & 43.3 & 142 & 3.3 & LS3 & Ade & pT1aNOMO & $\mathrm{IA}$ \\
\hline 2 & $70-79$ & 56.5 & 166 & 2.9 & R S6 & SCC & CT3N2MO & $\| \mathrm{A}$ \\
\hline 3 & $80-89$ & 50.0 & 239 & 4.8 & $L S 1+2$ & Ade & CT2aNOMO & $\mathrm{IB}$ \\
\hline 4 & $60-69$ & 46.3 & 162 & 3.5 & L S8 & SCC & pT2aNOMO & $\mathrm{IB}$ \\
\hline 5 & $80-89$ & 52.6 & 283 & 5.4 & R S7 & Ade & pT2aN2M0 & $\mathrm{IB}$ \\
\hline 6 & $60-69$ & 63.0 & 164 & 2.6 & R S6 & SCC & pT1aNOMO & IA \\
\hline 7 & $70-79$ & 48.6 & 146 & 3.0 & R S3 & Ade & pT1bNOMO & $\mathrm{IA}$ \\
\hline 8 & $70-79$ & 46.5 & 144 & 3.1 & R S2 & Ade & pT2aN1M0 & $\| \mathrm{A}$ \\
\hline 9 & 70-79 & 72.4 & 292 & 4.0 & R S5 & SCC & pT1aNOMO & $\mathrm{IA}$ \\
\hline 10 & 70-79 & 47.4 & 242 & 5.1 & R S1 & Ade & cT4N2M0 & $\| \mathrm{B}$ \\
\hline 11 & $30-39$ & 40.7 & 147 & 3.6 & R S8 & MEDC & pT1bNOMO & $\mathrm{IA}$ \\
\hline 12 & 70-79 & 45.9 & 294 & 6.4 & L S9 & Ade & pT1bNOMO & IA \\
\hline 13 & $50-59$ & 50.0 & 239 & 4.8 & R S1 & Ade & pT1aNOMO & $\mathrm{IA}$ \\
\hline 14 & $60-69$ & 65.7 & 276 & 4.2 & $L S 1+2$ & SCC & cT4N3M1b & IV \\
\hline 15 & $50-59$ & 60.7 & 294 & 4.8 & L S5 & Ade & pT2aN1M1b & IV \\
\hline 16 & $60-69$ & 52.1 & 243 & 4.7 & R S2 & SCC & cT4N3M0 & $\| \mathrm{B}$ \\
\hline 17 & $80-89$ & 56.2 & 236 & 4.2 & R S2 & Ade & pT1aNOMO & IA \\
\hline 18 & 70-79 & 57.4 & 145 & 2.5 & R S7 & LCNEC & pT2aN2M0 & $\mathrm{IB}$ \\
\hline 19 & 70-79 & 78.4 & 242 & 3.1 & $L S 1+2$ & SCC & pT1bNOMO & IA \\
\hline 20 & $50-59$ & 60.6 & 242 & 4.0 & R S1 & Ade & $\mathrm{pT} 1 \mathrm{bN} 2 \mathrm{M} 0$ & $\| \mathrm{A}$ \\
\hline 21 & $60-69$ & 67.2 & 243 & 3.6 & LS3 & Ade & pT3N1M0 & $\| \mathrm{A}$ \\
\hline 22 & $80-89$ & 48.5 & 240 & 5.0 & R S10 & Ade & pT2bNOMO & $\| \mathrm{A}$ \\
\hline 23 & $60-69$ & 64.7 & 243 & 3.8 & L S10 & SCC & pT3NOMO & $\| B$ \\
\hline 24 & 70-79 & 51.4 & 242 & 4.7 & LS9 & Ade & pT2bNOMO & $\| \mathrm{A}$ \\
\hline 25 & $60-69$ & 54.8 & 292 & 5.3 & R S3 & Ade & pT2aNOMO & $\| \mathrm{A}$ \\
\hline 26 & $60-69$ & 45.9 & 224 & 4.9 & $L S 1+2$ & Ade & pT4NOMO & $\| \mathrm{II}$ \\
\hline 27 & $80-89$ & 62.7 & 242 & 3.9 & $L S 1+2$ & SCC & cT3NOMO & $\| B$ \\
\hline 28 & 70-79 & 55.4 & 146 & 2.6 & R S4 & Ade & cT2bN1M1b & IV \\
\hline 29 & $60-69$ & 59.4 & 239 & 4.0 & R S2 & Ade & pT2bNOMO & $\| \mathrm{A}$ \\
\hline 30 & 70-79 & 53.0 & 145 & 2.7 & L S10 & $P C$ & pT2aN1M0 & $\| B$ \\
\hline 31 & 70-79 & 55.3 & 237 & 4.3 & R S1 & $P C$ & pT1aNOMO & $\mathrm{IA}$ \\
\hline 32 & 70-79 & 50.7 & 146 & 2.9 & L S4 & Ade & pT1aN3M0 & $\| \mathrm{B}$ \\
\hline 33 & $60-69$ & 68.8 & 274 & 4.0 & R S5 & Ade & cT2aN2M1 & IV \\
\hline 34 & $60-69$ & 57.2 & 245 & 4.3 & R S1 & SCC & cT3N2M0 & $\| \mathrm{A}$ \\
\hline 35 & 70-79 & 45.6 & 242 & 5.3 & R S1 & Ade & pT1aNOMO & $\mathrm{IA}$ \\
\hline 36 & $80-89$ & 54.6 & 274 & 5.0 & $L S 1+2$ & Ade & pT3N1M0 & $\| \mathrm{II}$ \\
\hline 37 & 70-79 & 46.6 & 146 & 3.1 & R S3 & Ade & $\mathrm{pT} 1 \mathrm{aN} 2 \mathrm{MO}$ & $\| \mathrm{A} A$ \\
\hline 38 & $80-89$ & 51.4 & 292 & 5.7 & R S10 & LCNEC & pT2aNOMO & $\| \mathrm{A}$ \\
\hline 39 & $80-89$ & 74.8 & 273 & 3.6 & R S4 & Ade & pT2aNOMO & $\| \mathrm{A}$ \\
\hline 40 & $60-69$ & 51.9 & 166 & 3.2 & R S3 & Ade & cT2aNOM1b & IV \\
\hline 41 & 70-79 & 40.8 & 164 & 4.0 & R S3 & Ade & pT2aNOMO & $\mathrm{IB}$ \\
\hline 42 & $50-59$ & 45.2 & 166 & 3.7 & LS3 & SCC & CT4N2MO & $\| \mathrm{B}$ \\
\hline 43 & $50-59$ & 61.4 & 266 & 4.3 & R S7 & $P C$ & pT2aN2M1 & IV \\
\hline 44 & $50-59$ & 72.2 & 290 & 4.0 & L S10 & $\mathrm{SCC}$ & $\mathrm{cT} 2 \mathrm{bN} 3 \mathrm{MO}$ & $\| \mathrm{B}$ \\
\hline
\end{tabular}


Table 1 Patient characteristics (Continued)

\begin{tabular}{lllllllll}
\hline 45 & $60-69$ & 62.4 & 166 & 2.7 & R S1 & LCNEC & pT2aNOM0 & IIA \\
46 & $60-69$ & 54.3 & 274 & 5.1 & R S8 & Ade & pT1aNOM0 & IA \\
47 & $70-79$ & 59.4 & 164 & 2.8 & R S3 & SCC & PT2aNOM0 & IB \\
48 & $60-69$ & 65.6 & 239 & 3.6 & LS3 & Ade & pT2aN1M0 & IIA \\
49 & $70-79$ & 61.9 & 292 & 4.7 & R S3 & SCC & PT1bN2M0 \\
50 & $60-69$ & 60.9 & 290 & 4.8 & R S6 & ASCC & PT1aN1M0 & IIA \\
\hline
\end{tabular}

${ }^{a}$ Ade adenocarcinoma, SCC squamous cell carcinoma, ASCC adenosquamous carcinoma, $P C$ pleomorphic carcinoma, MEDC mucoepidemoid carcinoma, $L C N E C$ large cell neuroendocrine carcinoma

The iteration and filter cycle values for DRAMA were 1 and 128 , respectively. The reconstructed image had a spatial resolution of $8.4 \mathrm{~mm}$ full-width at half-maximum and a matrix size of $128 \times 128$ with the voxel size $4.0 \times$ $4.0 \times 2.0 \mathrm{~mm}$. A smoothing filter was not applied.

\section{Image processing}

A total of 100 FDG-PET datasets (two datasets, i.e., early and delayed images, from 50 patients) were processed to delineate the tumor by two operators (Fig. 1). Operator1 (T.K.) is an experienced radiologic technologist of nuclear medicine, and Operator-2 (K.H.) is an experienced nuclear medicine physician. Both Operator- 1 and Operator-2 independently defined the tumor boundary two times with an interval of 30 days or longer (i.e., a total of 4-time measurements). Operator-2 defined the tumor boundary once without viewing the results reported by Operator-1, and vice versa. Hereinafter, we
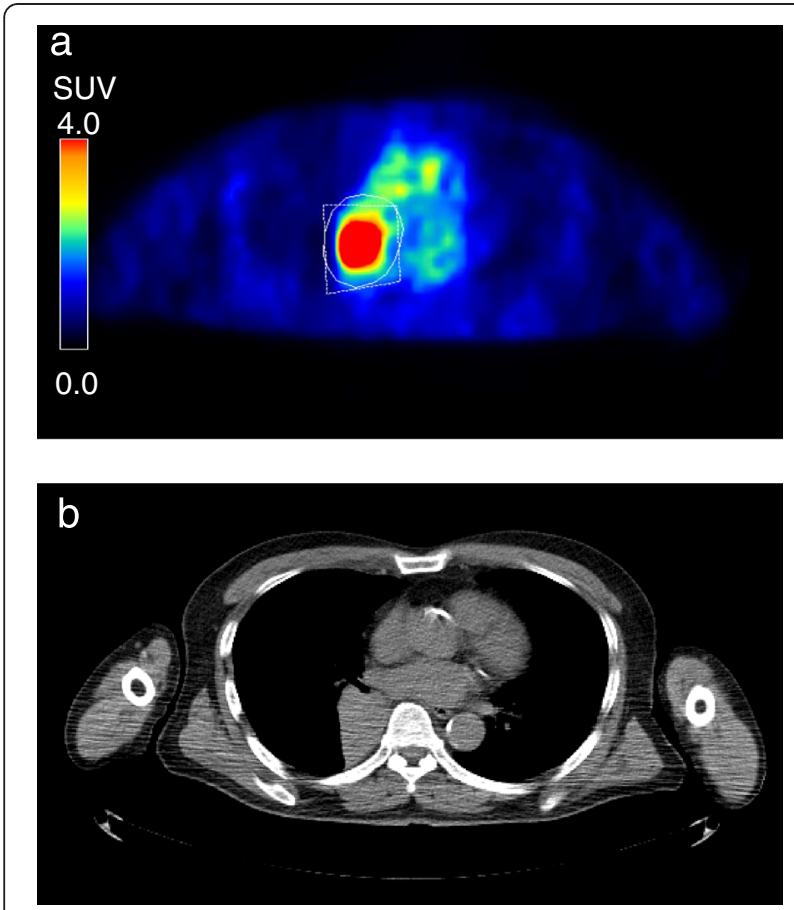

Fig. 1 a Regions of interest defined by Operator-1 (solid line) and Operator-2 (dashed line). b Corresponding CT slice use these three abbreviations: $\mathrm{Op}_{1} \mathrm{Ob}_{1}$ representing the first observation from operator-1, $\mathrm{Op}_{1} \mathrm{Ob}_{2}$ representing the second observation from operator- $1, \mathrm{Op}_{2} \mathrm{Ob}_{1}$ representing the first observation from Operator-2, and $\mathrm{Op}_{2} \mathrm{Ob}_{2}$ representing the second observation from Operator-2. The volume-of-interest (VOI) was defined by manually drawing polygonal regions of interest (ROIs) to enclose the entire tumor with enough margins on every slice where the tumor was seen. During the ROI definition, the PET images were displayed using a rainbow color bar with a fixed window level of SUV 04. Physiological uptake was carefully avoided. Neither lymph nodes nor distant metastatic lesions were investigated in this study. All of the ROIs were combined to generate a three-dimensional VOI.

In this study, we used the following three delineation methods. (1) The absolute SUV threshold method, which is a procedure of defining the area of the tumor as a region with a certain value higher than predetermined threshold, such as an SUV of 2.5 or 3.0. (2) The fixed\% SUVmax threshold method, which is a procedure for defining the area of the tumor as a region with a higher SUV than a certain percentage of the SUVmax within the tumor (40-50\%, commonly). (3) The adaptive regiongrowing method (ARG), which is a relatively new method [26]. The ARG is essentially a region-growing method that examines neighboring voxels of the current region and determines whether the neighbor voxels should be added to the in-tumor region. If $\{$ a neighbor voxel $\} \geq\{$ mean of current region $\} \times$ arbitrary threshold $\}$, the voxel is added to the region. There is a sharp volume increase point when the threshold (\%) varies from 100 to $0 \%$, and the tumor region is determined by this border point. With this method, the area of the tumor can be extracted automatically by the setting of the highest voxel in the tumor. Because the ARG method uses a new procedure, there are still few studies using this method.

The tumor volume was automatically determined within the VOI using different methods: $\mathrm{MTV}_{\mathrm{p}}, \mathrm{MTV}_{\mathrm{q} \%}$, and $\mathrm{MTV}_{\mathrm{ARG}}$. MTV $\mathrm{p}$ is the MTV determined using the absolute SUV threshold method, where $p=2.0,2.5,3.0$, or 3.5. $\mathrm{MTV}_{\mathrm{q} \%}$ is the MTV determined using the fixed\% SUVmax threshold method, where $q=35,40$, or $45 \%$. 
Values of $\mathrm{p}$ and $\mathrm{q}$ were chosen based on their frequency of appearance in literature [13]. $\mathrm{MTV}_{\mathrm{ARG}}$ is the MTV determined using ARG method.

TLG was defined as the product of the corresponding MTV and SUVmean values within the tumor boundary. The SUVmax was also recorded, which represented the voxel showing the highest SUV in the VOI. The SUV was calculated as [tissue radioactivity concentration $(\mathrm{Bq} /$ $\mathrm{ml})] \times$ [body weight $(\mathrm{g})] /[$ injected radioactivity $(\mathrm{Bq})]$.

For all the image analysis including manual ROI drawing, mathematical delineation, and parameter calculation, we used an in-house software package, composed with Visual Studio 2010 (Microsoft Corporation, Redmond, Washington, USA) and C\# language.

\section{Statistical analysis}

Values are expressed as the mean $\pm \mathrm{SD}$. The free statistical package $\mathrm{R}$ version 3.2.5 ( $\mathrm{R}$ Project, http:// cran.r-project.org) was used for all statistical analyses. A paired $t$-test was used if the values could be considered paired. The method of Holm was used to adjust the $P$-values for multiple comparisons. The intraclass correlation (ICC) was used to evaluate intraand inter-operator reproducibility [33]. Intra-operator reproducibility was estimated by 2 combinations: 1 ) $\mathrm{Op}_{1} \mathrm{Ob}_{1}$ vs. $\mathrm{Op}_{1} \mathrm{Ob}_{2}$, and 2) $\mathrm{Op}_{2} \mathrm{Ob}_{1}$ vs. $\mathrm{Op}_{2} \mathrm{Ob}_{2}$.
Inter-operator reproducibility can be estimated by 4 combinations: 1) $\mathrm{Op}_{1} \mathrm{Ob}_{1}$ vs. $\mathrm{Op}_{2} \mathrm{Ob}_{1}$, 2) $\mathrm{Op}_{1} \mathrm{Ob}_{1}$ vs. $\left.\mathrm{Op}_{2} \mathrm{Ob}_{2}, 3\right) \mathrm{Op}_{1} \mathrm{Ob}_{2}$ vs. $\mathrm{Op}_{2} \mathrm{Ob}_{1}$, and 4) $\mathrm{Op}_{1} \mathrm{Ob}_{2}$ vs. $\mathrm{Op}_{2} \mathrm{Ob}_{2}$. $P$-values $<0.05$ were considered as significant.

\section{Results}

\section{Reproducibility}

Both intra- and inter-operator reproducibility were extremely high at the early phase (Table 2 ) and the delayed phase (Table 3). The ICC between the first versus second measurement by Operator- 1 or Operator- 2 was $>0.99$ for any parameters. Similarly, the ICC between Operator-1 versus Operator- 2 was $>0.99$ for any parameters. Among the parameters, no difference was observed in SUVmax, $\mathrm{MTV}_{\mathrm{ARG}}$ or $\mathrm{TLG}_{\mathrm{ARG}}$ in any case (i.e., perfect match). Comparisons between methods revealed that most of the $\mathrm{MTV}_{\mathrm{q} \%}$ values were lower than those of $\mathrm{MTV}_{\mathrm{p}}$ or $\mathrm{MTV}_{\mathrm{ARG}}$.

\section{Parameter changes from the early phase to the delayed phase}

Parameter changes from early to delayed phases are summarized in Table 4. The SUVmax increased in 49 of the $50(98 \%)$ cases at the delayed phase compared to the early phase (early, $9.1 \pm 4.9$; delayed, $11.1 \pm 6.0$; $P<0.0001)$. The MTV changes depended on the

Table 2 Intra- and inter-operator reproducibility of PET parameters at the early phase

\begin{tabular}{|c|c|c|c|c|c|c|c|c|c|c|}
\hline & \multirow{2}{*}{$\begin{array}{l}\text { Op1Ob1 } \\
n=50\end{array}$} & \multirow[t]{2}{*}{ Op1Ob2 } & \multirow[t]{2}{*}{ Op2Ob1 } & \multirow[t]{2}{*}{ Op2Ob2 } & \multicolumn{2}{|c|}{$\begin{array}{l}\text { ICC intra-operator } \\
\text { reproducibility }\end{array}$} & \multicolumn{4}{|c|}{ ICC inter-operator reproducibility } \\
\hline & & & & & $\begin{array}{l}\text { Op1Ob1 vs. } \\
\text { Op1Ob2 }\end{array}$ & $\begin{array}{l}\text { Op2Op1 vs. } \\
\text { Op2Op2 }\end{array}$ & $\begin{array}{l}\text { Op1Ob1 vs. } \\
\text { Op2Ob1 }\end{array}$ & $\begin{array}{l}\text { Op1Ob1 vs. } \\
\text { Op2Ob2 }\end{array}$ & $\begin{array}{l}\text { Op1Ob2 vs. } \\
\text { Op2Ob1 }\end{array}$ & $\begin{array}{l}\text { Op1Ob2 vs. } \\
\text { Op2Ob2 }\end{array}$ \\
\hline$S U V_{\max }$ & $9.1 \pm 4.9$ & $9.1 \pm 4.9$ & $9.1 \pm 4.9$ & $9.1 \pm 4.9$ & 1 & 1 & 1 & 1 & 1 & 1 \\
\hline $\mathrm{MTV}_{2.0}$ & $55.0 \pm 100.6$ & $54.9 \pm 102.5$ & $52.8 \pm 96.6$ & $52.8 \pm 96.4$ & $>0.999$ & $>0.999$ & 0.997 & 0.997 & 0.997 & 0.997 \\
\hline $\operatorname{MTV}_{2.5}$ & $39.4 \pm 72.1$ & $39.3 \pm 72.5$ & $39.1 \pm 70.7$ & $38.9 \pm 70.4$ & $>0.999$ & $>0.999$ & $>0.999$ & $>0.999$ & $>0.999$ & 0.999 \\
\hline $\mathrm{MTV}_{3.0}$ & $29.7 \pm 51.7$ & $29.7 \pm 51.6$ & $29.8 \pm 51.3$ & $29.7 \pm 51.1$ & $>0.999$ & $>0.999$ & $>0.999$ & $>0.999$ & $>0.999$ & $>0.999$ \\
\hline $\mathrm{MTV}_{3.5}$ & $23.4 \pm 38.2$ & $23.4 \pm 38.1$ & $23.5 \pm 38.0$ & $23.4 \pm 37.8$ & $>0.999$ & $>0.999$ & $>0.999$ & $>0.999$ & $>0.999$ & $>0.999$ \\
\hline $\mathrm{MTV}_{35 \%}$ & $25.0 \pm 31.9$ & $24.3 \pm 31.7$ & $23.7 \pm 31.5$ & $23.7 \pm 31.5$ & 0.998 & $>0.999$ & 0.997 & 0.992 & 0.997 & 0.997 \\
\hline $\mathrm{MTV}_{40 \%}$ & $19.2 \pm 22.9$ & $18.9 \pm 22.8$ & $18.6 \pm 22.7$ & $18.6 \pm 22.7$ & 0.998 & $>0.999$ & 0.998 & 0.996 & 0.998 & 0.998 \\
\hline $\mathrm{MTV}_{45 \%}$ & $15.0 \pm 17.1$ & $14.9 \pm 17.1$ & $14.8 \pm 16.9$ & $14.8 \pm 16.9$ & $>0.999$ & 1 & $>0.999$ & 0.999 & $>0.999$ & $>0.999$ \\
\hline $\mathrm{MTV}_{\mathrm{ARG}}$ & $43.0 \pm 62.1$ & $43.0 \pm 62.1$ & $43.0 \pm 62.1$ & $43.0 \pm 62.1$ & 1 & 1 & 1 & 1 & 1 & 1 \\
\hline $\mathrm{TLG}_{2.0}$ & $225.2 \pm 365.2$ & $224.9 \pm 369.0$ & $220.4 \pm 354.5$ & $220.0 \pm 353.5$ & $>0.999$ & $>0.999$ & 0.999 & 0.999 & 0.999 & 0.999 \\
\hline $\mathrm{TLG}_{2.5}$ & $190.4 \pm 304.6$ & $190.1 \pm 305.5$ & $189.8 \pm 300.1$ & $189.2 \pm 298.9$ & $>0.999$ & $>0.999$ & $>0.999$ & $>0.999$ & $>0.999$ & $>0.999$ \\
\hline $\mathrm{TLG}_{3.0}$ & $164.0 \pm 253.7$ & $163.7 \pm 253.4$ & $164.4 \pm 251.8$ & $163.8 \pm 250.7$ & $>0.999$ & $>0.999$ & $>0.999$ & $>0.999$ & $>0.999$ & $>0.999$ \\
\hline $\mathrm{TLG}_{3.5}$ & $143.6 \pm 215.0$ & $143.4 \pm 214.7$ & $144.1 \pm 213.7$ & $143.6 \pm 212.9$ & $>0.999$ & $>0.999$ & $>0.999$ & $>0.999$ & $>0.999$ & $>0.999$ \\
\hline $\mathrm{TLG}_{35 \%}$ & $132.7 \pm 179.5$ & $131.8 \pm 179.7$ & $131.4 \pm 179.2$ & $131.3 \pm 179.1$ & $>0.999$ & $>0.999$ & $>0.999$ & $>0.999$ & $>0.999$ & $>0.999$ \\
\hline $\mathrm{TLG}_{40 \%}$ & $112.8 \pm 148.3$ & $112.3 \pm 148.3$ & $112.2 \pm 147.7$ & $112.1 \pm 147.7$ & $>0.999$ & $>0.999$ & $>0.999$ & $>0.999$ & $>0.999$ & $>0.999$ \\
\hline$T L G_{45 \%}$ & $96.2 \pm 124.7$ & $96.0 \pm 124.7$ & $96.0 \pm 123.9$ & $96.0 \pm 123.9$ & $>0.999$ & 1 & $>0.999$ & $>0.999$ & $>0.999$ & $>0.999$ \\
\hline $\mathrm{TLG}_{\text {ARG }}$ & $196.4 \pm 290.0$ & $196.4 \pm 290.0$ & $196.4 \pm 290.0$ & $196.4 \pm 290.0$ & 1 & 1 & 1 & 1 & 1 & 1 \\
\hline
\end{tabular}


Table 3 Intra- and inter-operator reproducibility of PET parameters at the delayed phase

\begin{tabular}{|c|c|c|c|c|c|c|c|c|c|c|}
\hline & \multirow{2}{*}{$\begin{array}{l}\text { Op1Ob1 } \\
n=50\end{array}$} & \multirow[t]{2}{*}{ Op1Ob2 } & \multirow[t]{2}{*}{ Op2Ob1 } & \multirow[t]{2}{*}{ Op2Ob2 } & \multicolumn{2}{|c|}{$\begin{array}{l}\text { ICC intra-operator } \\
\text { reproducibility }\end{array}$} & \multicolumn{4}{|c|}{ ICC inter-operator reproducibility } \\
\hline & & & & & $\begin{array}{l}\text { Op1Ob1 vs. } \\
\text { Op1Ob2 }\end{array}$ & $\begin{array}{l}\text { Op2Op1 vs. } \\
\text { Op2Op2 }\end{array}$ & $\begin{array}{l}\text { Op1Ob1 vs. } \\
\text { Op2Ob1 }\end{array}$ & $\begin{array}{l}\text { Op1Ob1 vs. } \\
\text { Op2Ob2 }\end{array}$ & $\begin{array}{l}\text { Op1Ob2 vs. } \\
\text { Op2Ob1 }\end{array}$ & $\begin{array}{l}\text { Op1Ob2 vs. } \\
\text { Op2Ob2 }\end{array}$ \\
\hline$S U V_{\max }$ & $11.1 \pm 6.0$ & $11.1 \pm 6.0$ & $11.1 \pm 6.0$ & $11.1 \pm 6.0$ & 1 & 1 & 1 & 1 & 1 & 1 \\
\hline $\operatorname{MTV}_{2.0}$ & $56.2 \pm 107.2$ & $56.2 \pm 107.9$ & $54.1 \pm 101.0$ & $54.7 \pm 101.8$ & $>0.999$ & $>0.999$ & 0.996 & 0.995 & 0.993 & 0.994 \\
\hline $\operatorname{MTV}_{2.5}$ & $41.9 \pm 79.6$ & $41.8 \pm 79.3$ & $41.6 \pm 77.6$ & $41.9 \pm 78.0$ & $>0.999$ & $>0.999$ & 0.999 & 0.998 & 0.997 & 0.997 \\
\hline MTV $_{3.0}$ & $33.1 \pm 60.9$ & $33.0 \pm 60.3$ & $33.5 \pm 60.6$ & $33.3 \pm 60.3$ & $>0.999$ & $>0.999$ & $>0.999$ & 0.998 & 0.998 & 0.998 \\
\hline MTV $_{3.5}$ & $27.3 \pm 48.2$ & $27.2 \pm 47.7$ & $27.5 \pm 47.8$ & $27.7 \pm 48.1$ & $>0.999$ & $>0.999$ & $>0.999$ & 0.998 & 0.998 & 0.998 \\
\hline $\mathrm{MTV}_{35 \%}$ & $21.0 \pm 29.0$ & $20.9 \pm 28.7$ & $20.5 \pm 28.7$ & $20.6 \pm 28.8$ & $>0.999$ & $>0.999$ & 0.998 & 0.997 & 0.996 & 0.997 \\
\hline $\mathrm{MTV}_{40 \%}$ & $16.6 \pm 21.2$ & $16.5 \pm 21.0$ & $16.4 \pm 21.0$ & $16.5 \pm 21.1$ & $>0.999$ & $>0.999$ & 0.999 & 0.999 & 0.998 & 0.999 \\
\hline $\mathrm{MTV}_{45 \%}$ & $13.2 \pm 15.7$ & $13.1 \pm 15.5$ & $13.1 \pm 15.5$ & $13.1 \pm 15.6$ & $>0.999$ & $>0.999$ & $>0.999$ & $>0.999$ & $>0.999$ & $>0.999$ \\
\hline $\mathrm{MTV}_{\mathrm{ARG}}$ & $50.4 \pm 76.2$ & $50.4 \pm 76.2$ & $50.4 \pm 76.2$ & $50.4 \pm 76.2$ & 1 & 1 & 1 & 1 & 1 & 1 \\
\hline $\mathrm{TLG}_{2.0}$ & $259.1 \pm 431.3$ & $258.6 \pm 430.8$ & $254.4 \pm 414.8$ & $256.8 \pm 418.8$ & $>0.999$ & $>0.999$ & 0.998 & 0.997 & 0.996 & 0.997 \\
\hline $\mathrm{TLG}_{2.5}$ & $227.2 \pm 372.8$ & $226.4 \pm 370.5$ & $226.4 \pm 365.4$ & $228.2 \pm 368.4$ & $>0.999$ & $>0.999$ & $>0.999$ & 0.998 & 0.998 & 0.998 \\
\hline$T L G_{3.0}$ & $203.4 \pm 325.8$ & $202.6 \pm 322.9$ & $205.4 \pm 324.8$ & $203.8 \pm 322.0$ & $>0.999$ & $>0.999$ & $>0.999$ & 0.999 & 0.998 & 0.998 \\
\hline $\mathrm{TLG}_{3.5}$ & $184.5 \pm 288.8$ & $183.8 \pm 286.3$ & $184.9 \pm 285.5$ & $186.5 \pm 288.3$ & $>0.999$ & $>0.999$ & $>0.999$ & 0.999 & 0.998 & 0.999 \\
\hline $\mathrm{TLG}_{35 \%}$ & $145.2 \pm 206.5$ & $144.5 \pm 204.8$ & $144.3 \pm 204.7$ & $145.4 \pm 206.3$ & $>0.999$ & $>0.999$ & $>0.999$ & $>0.999$ & 0.999 & 0.999 \\
\hline $\mathrm{TLG}_{40 \%}$ & $125.8 \pm 173.3$ & $125.2 \pm 171.9$ & $125.0 \pm 171.2$ & $126.0 \pm 172.6$ & $>0.999$ & $>0.999$ & $>0.999$ & $>0.999$ & 0.999 & $>0.999$ \\
\hline $\mathrm{TLG}_{45 \%}$ & $108.1 \pm 145.3$ & $107.6 \pm 144.1$ & $107.3 \pm 143.2$ & $108.1 \pm 144.3$ & $>0.999$ & $>0.999$ & $>0.999$ & $>0.999$ & $>0.999$ & $>0.999$ \\
\hline$T L G_{A R G}$ & $249.4 \pm 383.5$ & $249.4 \pm 383.5$ & $249.4 \pm 383.5$ & $249.4 \pm 383.5$ & 1 & 1 & 1 & 1 & 1 & 1 \\
\hline
\end{tabular}

ICC intra-class correlation, Op1Ob1 Operator-1's first observation, Op1Ob2 Operator-1's second observation, Op2Ob1 Operator-2's first observation, Op2Ob2 Operator-2's second observation

Table 4 The parameter changes from the early phase to the delayed phase

\begin{tabular}{|c|c|c|c|c|}
\hline & Early Phase (E) & Delayed Phase (D) & $\mathrm{D} / \mathrm{E}$ (mean) & $P$-value \\
\hline & \multicolumn{4}{|l|}{$n=50$} \\
\hline$S U V_{\max }$ & $9.1 \pm 4.9$ & $11.1 \pm 6.0$ & 1.22 & $<0.0001$ \\
\hline $\mathrm{MTV}_{2.0}$ & $55.0 \pm 100.6$ & $56.2 \pm 107.2$ & 1.02 & NS \\
\hline $\mathrm{MTV}_{2.5}$ & $39.4 \pm 72.1$ & $41.9 \pm 79.6$ & 1.06 & NS \\
\hline $\mathrm{MTV}_{3.0}$ & $29.7 \pm 51.7$ & $33.1 \pm 60.9$ & 1.11 & $<0.05$ \\
\hline $\mathrm{MTV}_{3.5}$ & $23.4 \pm 38.2$ & $27.3 \pm 48.2$ & 1.17 & $<0.05$ \\
\hline $\mathrm{MTV}_{35 \%}$ & $25.0 \pm 31.9$ & $21.0 \pm 29.0$ & 0.84 & $<0.001$ \\
\hline $\mathrm{MTV}_{40 \%}$ & $19.2 \pm 22.9$ & $16.6 \pm 21.2$ & 0.86 & $<0.001$ \\
\hline $\mathrm{MTV}_{45 \%}$ & $15.0 \pm 17.1$ & $13.2 \pm 15.7$ & 0.88 & $<0.001$ \\
\hline $\mathrm{MTV}_{\mathrm{ARG}}$ & $43.0 \pm 62.1$ & $50.4 \pm 76.2$ & 1.17 & $<0.05$ \\
\hline $\mathrm{TLG}_{2.0}$ & $225.2 \pm 365.2$ & $259.1 \pm 431.3$ & 1.15 & $<0.01$ \\
\hline $\mathrm{TLG}_{2.5}$ & $190.4 \pm 304.6$ & $227.2 \pm 372.8$ & 1.19 & $<0.001$ \\
\hline $\mathrm{TLG}_{3.0}$ & $164.0 \pm 253.7$ & $203.4 \pm 325.8$ & 1.24 & $<0.001$ \\
\hline $\mathrm{TLG}_{3.5}$ & $143.6 \pm 215.0$ & $184.5 \pm 288.8$ & 1.28 & $<0.001$ \\
\hline $\mathrm{TLG}_{35 \%}$ & $132.7 \pm 179.5$ & $145.2 \pm 206.5$ & 1.09 & $<0.05$ \\
\hline $\mathrm{TLG}_{40 \%}$ & $112.8 \pm 148.3$ & $125.8 \pm 173.3$ & 1.12 & $<0.01$ \\
\hline $\mathrm{TLG}_{45 \%}$ & $96.2 \pm 124.7$ & $108.1 \pm 145.3$ & 1.12 & $<0.01$ \\
\hline$T L G_{A R G}$ & $196.4 \pm 290.0$ & $249.4 \pm 383.5$ & 1.27 & $<0.001$ \\
\hline
\end{tabular}

delineation methods. Among them, the $\mathrm{MTV}_{2.0}$ and $\mathrm{MTV}_{2.5}$ neither increased nor decreased from the early phase to the delayed phase with the averaged delayed-toearly ratios of 1.02 and 1.06 , respectively $(P=$ nonsignificant for both). The use of a higher threshold (i.e., $\mathrm{MTV}_{3.0}$ and $\left.\mathrm{MTV}_{3.5}\right)$ led to a significant increase from the early to the delayed phase $\left(P<0.05\right.$ for both). All of the $\mathrm{MTV}_{\mathrm{q} \%}$ values (i.e., $\mathrm{MTV}_{35 \%}, \mathrm{MTV}_{40 \%}$, and $\mathrm{MTV}_{45 \%}$ ) significantly decreased $(P<0.001)$, whereas the MTV $_{\mathrm{ARG}}$ values significantly increased $(P<0.05)$ (Fig. 2$)$. In contrast, the TLG obtained by any of the delineation methods was significantly increased at the delayed phase (Fig. 3).

\section{Discussion}

In this study of volume-based parameters on FDG-PET for NSCLC, we found high intra- and inter-operator reproducibility for all parameters (ICC $>0.99$ each). We also evaluated the time sensitivity of the parameters by comparing early-phase images with delayed-phase images. Whereas the SUVmax increased significantly at the delayed phase, the MTV changes depended on the delineation method, and the TLG obtained by any of the delineation methods was significantly increased at the delayed phase $(P<0.05)$. Among the parameters examined, only $\mathrm{MTV}_{2.0}$ and $\mathrm{MTV}_{2.5}$ were the parameters that neither increased nor decreased at the delayed phase. 


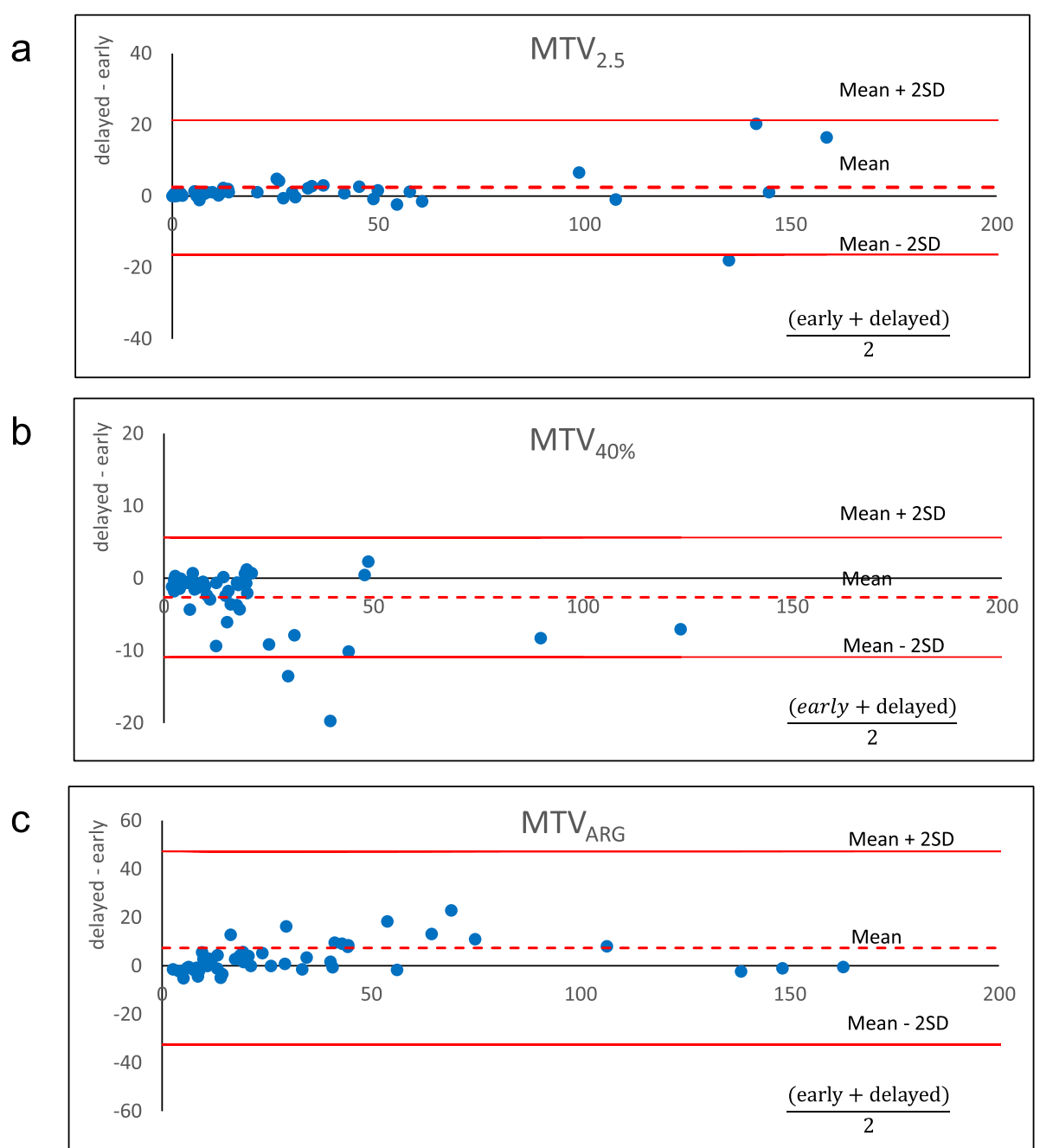

Fig. 2 Bland-Altman plots showing the parameter changes between the early phase and delayed phase of the MTV, which is a general threshold

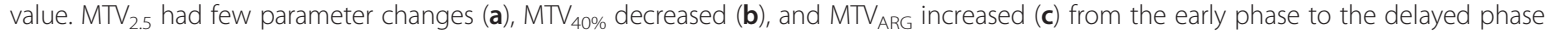

\section{Intra- and inter-operator reproducibility}

In case that the tumor exists without adjacent nontumor uptakes (i.e., physiological or inflammatory), the semi-automated methods we employed in this study should not cause variability of measurement theoretically. However, it is not uncommon that the tumor is so close to mediastinum that the manual ROIs include parts of blood pool or lymph nodes. In such cases, even semi-automated methods are expected to cause some variation if the threshold is lower than the non-tumor uptake. In this study, we observed both the intra- and inter-operator reproducibility were high for all parameters. Although we observed minimal differences in some cases between the two measurements when relatively low threshold (absolute or fixed\% SUVmax) was used, as expected, we consider that the high ICCs may allow use of the methods. Shah et al. reported high inter-operator reproducibility of MTV and TLG using a fixed\%
SUVmax threshold method that showed the ICCs between two measurements by one operator as $>0.98$ for MTV and $>0.99$ for TLG [33]. Frings et al. demonstrated high repeatability in the same examination of the two measurement within 1 week using FDG or 18Ffluorothymidine (FLT) [34]. Our results are in line with these previous reports. The difference we observed may be small enough for clinical use.

In contrast, using the ARG method, the twicemeasurements of the tumor volume completely agreed, because this method delineates the tumor boundary without requiring a manual ROI [26]. Our results are consistent with this report in terms of high interoperator reproducibility. However, as a shortcoming, this method does not always successfully determine the tumor boundary, especially when images are noisy or the boundaries are indistinct (or ambiguous). Conducting phantom experiments, $\mathrm{Li}$ et al. reported that the 

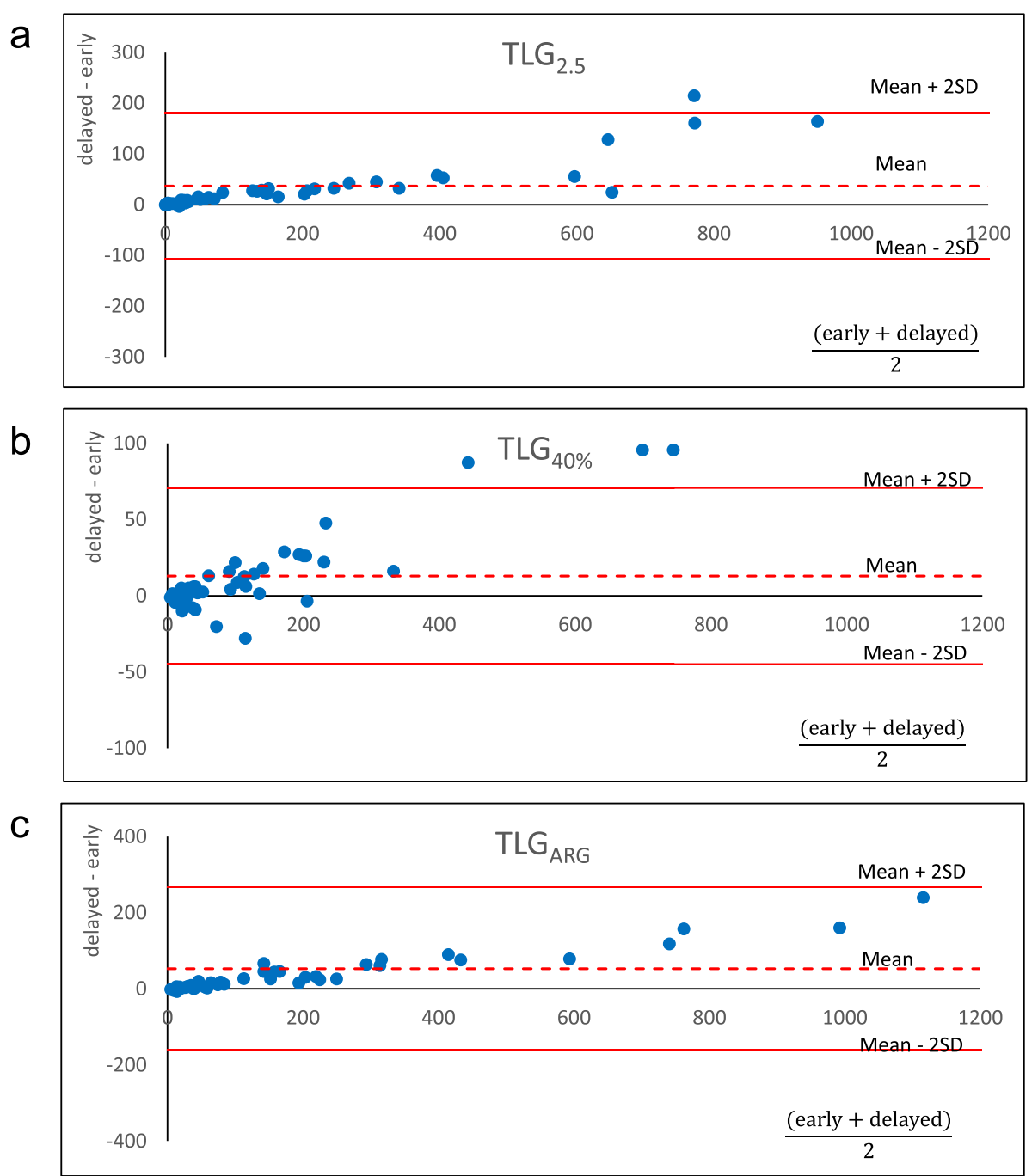

Fig. 3 Bland-Altman plots showing the parameter changes between the early phase and the delayed phase of TLG, which is general threshold value. Unlike Fig. 2, TLG obtained by any delineation method was increased at the delayed phase (a-c)

ARG method generates a slightly larger volume than the actual tumor volume, and that the degree of volume overestimation depended on the source-to-background ratio. They thus recommended that use of the ARG method should be followed by an appropriate volume correction.

\section{Early and delayed scans}

MTV is the volume where the tumor cells are actively metabolizing glucose. Note that MTV is not an uptake quantification. The volume should not change within a few hours but should be stable if there is no significant tumor growth. In fact, however, many methods of MTV measurement resulted in significant volume changes from the early phase to the delayed phase except for $\mathrm{MTV}_{2.0}$ and $\mathrm{MTV}_{2.5}$. In contrast, TLG is the arbitrary amount of glucose metabolized during the period from injection to image acquisition. Thus, TLG may change over time theoretically. In the present study, we investigated malignant tumors only; thus, the FDG inflow is thought to continue even $1 \mathrm{~h}$ after the injection, resulting in higher uptake at $2 \mathrm{~h}[35,36]$. Among the MTVs measured by different methods, $\mathrm{MTV}_{2.0}$ and $\mathrm{MTV}_{2.5}$ neither increased nor decreased from the early to the delayed phase, probably because the increase in tumor uptake and the decrease in the surrounding background uptake (e.g., in a lung field or mediastinum blood pool) would have cancelled each other out. Conversely, the $\mathrm{MTV}_{35 \%}, \mathrm{MTV}_{40 \%}$, and $\mathrm{MTV}_{45 \%}$ values all significantly decreased because the increase in the SUVmax raised the delineation cut-off value. $\mathrm{MTV}_{\mathrm{ARG}}$ increased due to the increase in the tumor-to-background ratio at the delayed phase. TLG by all delineation methods significantly increased; this is likely due to the increase in the 
SUVmean within the region. Our present report is the first to show parameter changes from the early to delayed phases.

PERCIST, the guideline for PET response criteria in solid tumors, requires that a PET scan for baseline should be obtained at 50-70 min after injection, and the follow-up scan should be obtained within $15 \mathrm{~min}$ of the baseline scan [13]. In our observation, almost all parameters changed from the early phase to the delayed phase, which further supported the importance of time strictness. However, it is not always easy to perform scanning under such a strict protocol in many clinical conditions. In particular, when we try to carry out a retrospective analysis, the uptake time restriction will exclude a number of scans. We suggest that use of $\mathrm{MTV}_{2.0}$ or $\mathrm{MTV}_{2.5}$ could be an alternative way to minimize the influence of uptake time variability.

It should be noted that $\mathrm{MTV}_{2.5}$ is the most commonly used method thus far, and is known to be well correlated with patient outcomes of various cancers [27, 28, 37]. For instance, Kao et al. showed that $\mathrm{MTV}_{2.5}$ was the most appropriate parameter for predicting recurrence after radiotherapy for pharyngeal cancer patients in comparison with $\mathrm{MTV}_{3.0}, \mathrm{MTV}_{40 \%}$, and $\mathrm{MTV}_{50 \%}$ [28]. Based on our present findings, $\mathrm{MTV}_{3.0}$ or MTV with higher thresholds may not be appropriate if the uptake time is not constant. Another reason to avoid higher thresholds is that a significant number of cases showed zero volume using such thresholds.

$\mathrm{MTV}_{\mathrm{q} \%}$ has also been frequently used. MTV $\mathrm{M}_{\mathrm{q} \%}$ is actually better at tumor volume measurements in a phantom study because it is relatively resistant to partial volume effects. However, this method may appropriately work when the tumor has intermediate SUVmax (e.g., 5-10) but may under- or over-estimate the volume in cases of considerably high or low SUVmax of tumor, respectively. Therefore, it is difficult to fix relative threshold (\%) in studies investigating a large number of patients. Considering the difficulty in fixing an absolute or relative SUV threshold, the ARG procedure is an attractive method that does not require manual interaction. Although the ARG method did achieve very good intra- and inter-operator reproducibility in the present study, its high sensitivity to uptake time necessitated further improvement. TLG seems to extract more information of PET than MTV does, because TLG is an uptake quantification whereas MTV is just a volume. Superiority of TLG to MTV for treatment response of lung cancer has been reported recently [38, 39]. As mentioned above, however, the TLG obtained by any of the delineation methods was significantly increased at the delayed phase. Therefore, when we use datasets acquired with a fluctuating uptake time, we recommend that $\mathrm{MTV}_{2.5}$ should be chosen as the best volume-based parameter among many MTVs and TLGs.
The limitations of this study include the following. We investigated reproducibility and parameter changes by uptake time, but we did not report the prognostic value. Future studies will be needed to combine the present findings and prognostic information. In addition, it is necessary to study cancers other than lung cancer. For lung cancer, a manual ROI was defined relatively easily because the tumor existed in the lung showing low FDG uptake. Reproducibility may be affected in fields that have higher physiological uptake, such as the head-andneck and pelvis.

\section{Conclusions}

The MTV and TLG of primary lesions of 50 NSCLC patients were measured with different tumor delineation methods and different uptake times. We found that both the intra- and inter-operator reproducibility were extremely high for all parameters. Most of the MTV values and all of the TLG values were significantly affected by the uptake time. Among the various parameters studied, $\mathrm{MTV}_{2.0}$ and $\mathrm{MTV}_{2.5}$ were the least sensitive to the uptake time, and may be good alternatives when we compare images acquired with different uptake times, although applying constant uptake time is important for volume measurement.

\section{Abbreviations \\ $\mathrm{CT}$, computed tomography; DRAMA, dynamic row-action maximum likeli- hood algorithm; FDG, fluorodeoxyglucose; FLT, fluorothymidine; ICC, intra- class correlation; ICCop1, ICC between the first versus second measurement by operator-1; ICCop1op2, ICC between operator-1 versus operator-2; MRI, magnetic resonance imaging; MTV, metabolic tumor volume; NSCLC, non- small cell lung cancer; PET, positron emission tomography; RAMLA, row- action maximum likelihood algorithm; ROIs, regions of interest; SUV, stan- dardized uptake value; SUVmax, maximum of SUV; TLG, total lesion glycolysis; VOI, volume-of-interest}

\section{Acknowledgements}

We thank Eriko Suzuki for her persistent support in the preparation of the manuscript. This study was funded by Foundation for Promotion of Cancer Research in Japan.

\section{Funding}

This study was funded by Foundation for Promotion of Cancer Research in Japan.

\section{Availability of data and materials}

The datasets supporting the conclusions of this article are included within the article.

\section{Authors' contributions}

TK and KH collected the data, performed the analysis, and wrote the manuscript. NT participated in the design of the study and helped draft the manuscript. KS, TH, and MS helped collect the data. WI and TT provided technical assistance. $\mathrm{MH}$ and $\mathrm{KK}$ helped edit the manuscript. All authors read and approved the final manuscript.

\section{Competing interests}

The authors declare that they have no competing interests.

Consent for publication

Not applicable. 


\section{Ethics approval and consent to participate}

This retrospective study was approved by the institutional ethics committee of Hokkaido Cancer Center (Approval number: 25-24). The informed consent was waived from individual participants in the retrospective study according to the institutional ethics committee of Hokkaido Cancer Center. Patient records/information was anonymized and de-identified prior to analysis.

\section{Statement of human rights}

All procedures performed in studies involving human participants were in accordance with the ethical standards of the institutional and/or national research committee and with the 1964 Helsinki declaration and its later amendments or comparable ethical standards.

\section{Author details}

${ }^{1}$ Radiology Department, National Hospital Organization, Hokkaido Cancer Center, 2-3-54, Kikusui-4, Shiroishi-Ku, Sapporo 003-0804, Japan. ${ }^{2}$ Department of Nuclear Medicine, Graduate School of Medicine, Hokkaido University, Kita 15, Nishi 7, Kita-Ku, Sapporo, Hokkaido 060-8638, Japan. ${ }^{3}$ Department of Diagnostic Radiology, Hokkaido Cancer Center, Sapporo, Japan. ${ }^{4}$ Department of Respiratory Medicine, Hokkaido Cancer Center, Sapporo, Japan. ${ }^{5}$ Department of Thoracic Surgery, Hokkaido Cancer Center, Sapporo, Japan.

Received: 9 February 2016 Accepted: 26 July 2016

\section{Published online: 02 August 2016}

\section{References}

1. Ben-Haim S, Ell P. 18F-FDG PET and PET/CT in the evaluation of cancer treatment response. J Nucl Med. 2009;50(1):88-99.

2. Jhanwar YS, Straus DJ. The role of PET in lymphoma. J Nucl Med. 2006;47(8): 1326-34.

3. Israel O, Kuten A. Early detection of cancer recurrence: 18 F-FDG PET/CT can make a difference in diagnosis and patient care. J Nucl Med. 2007:48 Suppl 1:28S-35.

4. Inoue $T$, Kim EE, Komaki R, Wong FC, Bassa P, Wong WH, et al. Detecting recurrent or residual lung cancer with FDG-PET. J Nucl Med. 1995;36(5):788-93.

5. Wu H, Dimitrakopoulou-Strauss A, Heichel TO, Lehner B, Bernd L, Ewerbeck $V$, et al. Quantitative evaluation of skeletal tumours with dynamic FDG PET: SUV in comparison to Patlak analysis. Eur J Nucl Med. 2001;28(6):704-10.

6. Adams S, Baum RP, Stuckensen T, Bitter K, Hor G. Prospective comparison of 18 F-FDG PET with conventional imaging modalities (CT, MRI, US) in lymph node staging of head and neck cancer. Eur J Nucl Med. 1998;25(9):1255-60.

7. Thie JA. Understanding the standardized uptake value, its methods, and implications for usage. J Nucl Med. 2004:45(9):1431-4.

8. Higashi K, Ueda Y, Arisaka Y, Sakuma T, Nambu Y, Oguchi M, et al. 18 F-FDG uptake as a biologic prognostic factor for recurrence in patients with surgically resected non-small cell lung cancer. J Nucl Med. 2002:43(1):39-45.

9. Higashi K, Matsunari I, Ueda Y, Ikeda R, Guo J, Oguchi M, et al. Value of whole-body FDG PET in management of lung cancer. Ann Nucl Med. 2003; 17(1):1-14.

10. Hicks RJ, Kalff V, MacManus MP, Ware RE, Hogg A, McKenzie AF, et al. (18)F-FDG PET provides high-impact and powerful prognostic stratification in staging newly diagnosed non-small cell lung cancer. J Nucl Med. 2001; 42(11):1596-604

11. Gebhart G, Gamez C, Holmes E, Robles J, Garcia C, Cortes M, de Azambuja E, et al. 18 F-FDG PET/CT for early prediction of response to neoadjuvant lapatinib, trastuzumab, and their combination in HER2-positive breast cancer: results from Neo-ALTTO. J Nucl Med. 2013:54(11):1862-8.

12. Hanin FX, Lonneux M, Cornet J, Noirhomme P, Coulon C, Distexhe J, et al. Prognostic value of FDG uptake in early stage non-small cell lung cancer. Eur J Cardiothorac Surg. 2008;33(5):819-23.

13. Wahl RL, Jacene $H$, Kasamon $Y$, Lodge MA. From RECIST to PERCIST: Evolving Considerations for PET response criteria in solid tumors. J NuCl Med. 2009;50 Suppl 1:122S-50.

14. Lodge MA, Chaudhry MA, Wahl RL. Noise considerations for PET quantification using maximum and peak standardized uptake value. J Nucl Med. 2012;53(7):1041-7.

15. Vanderhoek M, Perlman SB, Jeraj R. Impact of different standardized uptake value measures on PET-based quantification of treatment response. J Nucl Med. 2013;54(8):1188-94
16. Liao S, Penney BC, Zhang H, Suzuki K, Pu Y. Prognostic value of the quantitative metabolic volumetric measurement on 18 F-FDG PET/CT in Stage IV nonsurgical small-cell lung cancer. Acad Radiol. 2012;19(1):69-77.

17. Chen HH, Chiu NT, Su WC, Guo HR, Lee BF. Prognostic value of whole-body total lesion glycolysis at pretreatment FDG PET/CT in non-small cell lung cancer. Radiology. 2012;264(2):559-66.

18. Ryu IS, Kim JS, Roh JL, Lee JH, Cho KJ, Choi SH, et al. Prognostic value of preoperative metabolic tumor volume and total lesion glycolysis measured by 18 F-FDG PET/CT in salivary gland carcinomas. J NuCl Med. 2013;54(7):1032-8.

19. Chu KP, Murphy JD, La TH, Krakow TE, lagaru A, Graves EE, et al. Prognostic value of metabolic tumor volume and velocity in predicting head-and-neck cancer outcomes. Int J Radiat Oncol Biol Phys. 2012;83(5):1521-7.

20. Abgral R, Keromnes N, Robin P, Le Roux PY, Bourhis D, Palard X, et al. Prognostic value of volumetric parameters measured by 18 F-FDG PET/CT in patients with head and neck squamous cell carcinoma. Eur J Nucl Med Mol Imaging. 2014;41(4):659-67.

21. Kidd EA, Thomas M, Siegel BA, Dehdashti F, Grigsby PW. Changes in cervical cancer FDG uptake during chemoradiation and association with response. Int J Radiat Oncol Biol Phys. 2013;85(1):116-22.

22. Liao S, Lan X, Cao G, Yuan H, Zhang Y. Prognostic predictive value of total lesion glycolysis from 18 F-FDG PET/CT in post-surgical patients with epithelial ovarian cancer. Clin Nucl Med. 2013;38(9):715-20.

23. Roedl JB, Colen RR, Holalkere NS, Fischman AJ, Choi NC, Blake MA. Adenocarcinomas of the esophagus: response to chemoradiotherapy is associated with decrease of metabolic tumor volume as measured on PET-CT. Comparison to histopathologic and clinical response evaluation. Radiother Oncol. 2008:89(3):278-86.

24. Cook GJ, Yip C, Siddique M, Goh V, Chicklore S, Roy A, et al. Are pretreatment 18 F-FDG PET tumor textural features in non-small cell lung cancer associated with response and survival after chemoradiotherapy? J Nucl Med. 2013:54(1):19-26.

25. Uto F, Shiba E, Onoue S, Yoshimura H, Takada M, Tsuji Y, et al. Phantom Study on Radiotherapy Planning Using PET/CT. J Radiat Res. 2010;51(2):157-64

26. Li H, Thorstad WL, Biehl KJ, Laforest R, Su Y, Shoghi Kl, et al. A novel PET tumor delineation method based on adaptive region-growing and dualfront active contours. Med Phys. 2008;35(8):3711-21.

27. Van de Wiele C, Kruse V, Smeets P, Sathekge M, Maes A. Predictive and prognostic value of metabolic tumour volume and total lesion glycolysis in solid tumours. Eur J Nucl Med Mol Imaging. 2013;40(2):290-301.

28. Kao CH, Lin SC, Hsieh TC, Yen KY, Yang SN, Wang YC, et al. Use of pretreatment metabolic tumour volumes to predict the outcome of pharyngeal cancer treated by definitive radiotherapy. Eur J Nucl Med Mol Imaging. 2012;39(8):1297-305.

29. Soussan M, Chouahnia K, Maisonobe JA, Boubaya M, Eder V, Morere JF, et al. Prognostic implications of volume-based measurements on FDG PET/ $C T$ in stage III non-small-cell lung cancer after induction chemotherapy. Eur J Nucl Med Mol Imaging. 2013;40(5):668-76.

30. Tahari AK, Alluri KC, Quon H, Koch W, Wahl RL, Subramaniam RM. FDG PET/ CT Imaging of Oropharyngeal Squamous Cell Carcinoma Characteristics of Human Papillomavirus-Positive and -Negative Tumors. Clin Nucl Med. 2014; 39(3):225-31.

31. Chen SW, Chen WTL, Wu YC, Yen KY, Hsieh TC, Lin TY, et al. Which FDG/PET parameters of the primary tumors in colon or sigmoid cancer provide the best correlation with the pathological findings? Eur J Radiol. 2013;82(9):E405-10.

32. Tanaka $\mathrm{E}$, Kudo $\mathrm{H}$. Subset-dependent relaxation in block-iterative algorithms for image reconstruction in emission tomography. Phys Med Biol. 2003; 48(10):1405-22

33. Shah B, Srivastava $N$, Hirsch AE, Mercier G, Subramaniam RM. Intrareader reliability of FDG PET volumetric tumor parameters: effects of primary tumor size and segmentation methods. Ann Nucl Med. 2012; 26(9):707-14

34. Frings V, de Langen AJ, Smit EF, van Velden FH, Hoekstra OS, van Tinteren $\mathrm{H}$, et al. Repeatability of metabolically active volume measurements with 18 F-FDG and 18 F-FLT PET in non-small cell lung cancer. J Nucl Med. 2010; 51(12):1870-7.

35. Cheng G, Torigian DA, Zhuang $H$, Alavi A. When should we recommend use of dual time-point and delayed time-point imaging techniques in FDG PET? Eur J Nucl Med Mol Imaging. 2013;40(5):779-87. 
36. Suga K, Kawakami Y, Hiyama A, Sugi K, Okabe K, Matsumoto T, Ueda K, Tanaka N, Matsunaga N. Dual-time point 18 F-FDG PET/CT scan for differentiation between 18 F-FDG-avid non-small cell lung cancer and benign lesions. Ann Nucl Med. 2009:23(5):427-35.

37. Byun BH, Kong CB, Park J, Seo Y, Lim I, Choi CW, et al. Initial metabolic tumor volume measured by 18 F-FDG PET/CT can predict the outcome of osteosarcoma of the extremities. J Nucl Med. 2013;54(10):1725-32.

38. Moon SH, Cho SH, Park LC, Ji JH, Sun JM, Ahn JS, et al. Metabolic response evaluated by 18 F-FDG PET/CT as a potential screening tool in identifying a subgroup of patients with advanced non-small cell lung cancer for immediate maintenance therapy after first-line chemotherapy. Eur J Nucl Med Mol Imaging. 2013;40(7):1005-13.

39. Usmanij EA, de Geus-Oei LF, Troost EG, Peters-Bax L, van der Heijden EH, Kaanders JH, et al. 18F-FDG PET early respons evaluation of locally advanced non-small cell lung cancer treated with concomitant chemoradiotherapy. J Nucl Med. 2013;54(9):1528-34.

Submit your next manuscript to BioMed Central and we will help you at every step:

- We accept pre-submission inquiries

- Our selector tool helps you to find the most relevant journal

- We provide round the clock customer support

- Convenient online submission

- Thorough peer review

- Inclusion in PubMed and all major indexing services

- Maximum visibility for your research

Submit your manuscript at www.biomedcentral.com/submit 